\title{
Multi-instrumented dynamic loading experiments on laminated glass
}

\author{
Corentin Le Gourriérec ${ }^{1,2 *}$, Bastien Durand ${ }^{1}$, Stéphane Roux ${ }^{1}$, Xavier Brajer ${ }^{2}$, Benô̂t \\ Voillot $^{2}$, and Richard Villey ${ }^{3}$ \\ ${ }^{1}$ Université Paris-Saclay, ENS Paris-Saclay, CNRS, LMT, 91190, Gif-sur-Yvette, France \\ ${ }^{2}$ Saint-Gobain Research Paris, 39 Quai Lucien Lefranc, 93303, Aubervilliers, France \\ ${ }^{3}$ Saint-Gobain Research Compiègne, 1 rue de Montluçon, 60777, Thourotte, France
}

\begin{abstract}
Describing quantitatively the response of laminated glass to low-velocity $(\sim 5 \mathrm{~m} / \mathrm{s})$ impacts by rigid bodies is an important issue because of its significance in terms of structural degradation and integrity, key parameters for people safety and anti-intrusion performances. This study aims to address the formation of cracks during graveling and steel ball drop tests, so, two well-instrumented experimental set-ups are proposed to study cracking in reproducible conditions. The first device can be seen as a mini-Hopkinson bar system, which from two strain gauges, allows to estimate force and velocity at a sharp indent tip. The second device, reproducing a blunt impact, exploits stereo-Digital Image Correlation (D.I.C.) measurements of the laminated glass surface.
\end{abstract}

\section{Introduction}

Laminated glass is a composite material, built with two or more glass plies, interleaved with polymer layers. In this study, its structure combines flat soda-lime glass, a brittle and rigid material, with polyvinyl butyral (PVB), a soft and viscoelastic thermoplastic which can withstand large strains. The laminated glass behavior is complex, and its impact performance depends on many parameters. The adhesion between PVB and glass sheets is one of the most significant parameters in terms of performance with respect to users safety. Low adhesion could lead to large glass chips splintering during impact, but too high adhesion prevents a good dissipation of impact energy, leading to tearing of the polymer layer. To optimize laminated glasses, a large number of experiments in different configurations are needed. Moreover, to certify their products, the glass manufacturers must pass normalized tests (e.g. European norms for building application, EN 356 and EN 12600). They aim to evaluate the re-

\footnotetext{
*Corresponding author: corentin.le_gourrierec-braun@ens-paris-saclay.fr
} 
sistance of laminates under common accidental situations, by reproducing for instance stone impact on a windshield or a window break-in for building applications.

As a result, instrumented tests are developed to replicate the mechanisms involved during an impact on laminated glass. Two set-ups will be presented afterwards: the "blowpipe" test, allowing to reproduce the formation of small star cracks specific to the projection of a gravel on a windscreen [1], i.e. a gravel test. As well as the "cannon" test, designed to reproduce instrumented experiments inspired by the norm EN 356 on a test bench [2, 3], and whose concept is adapted to new measurements.

\section{2 "Blowpipe" experiments}

Impact tests are carried out in the automotive industry to validate the impact resistance of windscreens. Numerous sharp indentations are repetitively performed on the external glass surface for a statistical analysis, allowing for laminate optimization. In ref. [1], Grant studied the phenomenological aspects of graveling and concluded that crack formation strongly depends on test parameters (impact velocity, angle, gravel mass) but also on the glass sample geometry and on the boundary conditions. During gravel testing, the polymer interlayer improves the laminated glass performance as compared to monolithic glass samples. However, an accurate identification of the influence of each ply and interface is difficult in particular because of the needed statistical analysis. Thus, more reproducible and instrumented experiments would be highly beneficial.

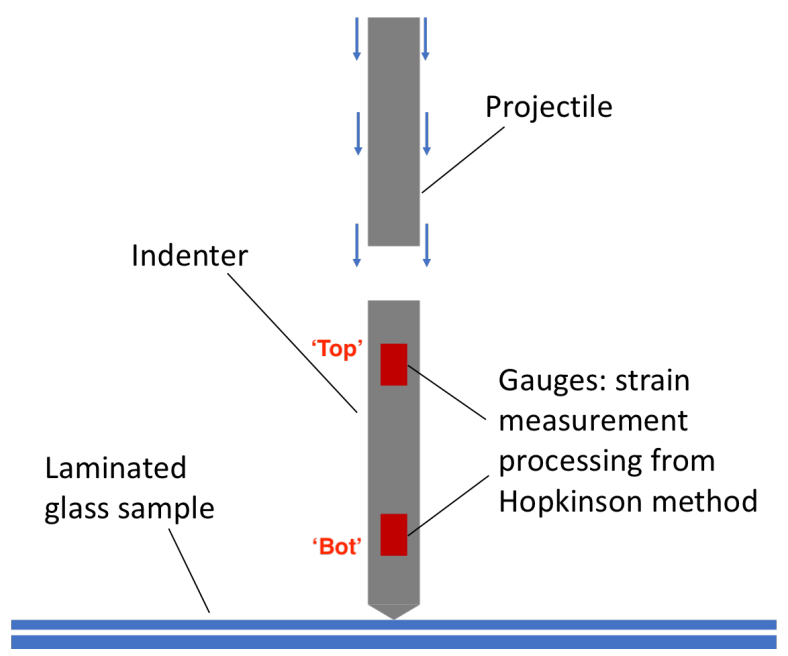

Fig. 1. Scheme of a newly designed "blowpipe" experiment. It includes an initially moving projectile and an initially motionless indent placed on the external glass surface. Two gauge locations are represented: the 'top' gauge, closer to the projectile and the bottom ('bot') gauge closer to the indent tip.

The "blowpipe" test design represented on Fig. 1, is derived from the gravel tests performed 
to evaluate the laminated glass windshield resistance. This device was first developed to study the initiation process of star-cracks, but has then been adapted to analyze the crack propagation and arrest, and also to finally describe the crack patterns versus emphasized key parameters such as the impact velocity or the glass ply thicknesses. An indent set on the external layer surface is impacted by a $4 \mathrm{~g}$ projectile at $4 \mathrm{~m} / \mathrm{s}$. The steel indent and its synthetic diamond $200 \mu \mathrm{m}$ Rockwell tip are supposed to remain purely elastic. The indent displacements being small, strain gauges can be glued at 2 locations along its length, and a SHPB-like analysis [4] is performed to measure the impact velocity and force next to the glass surface. Due to the length/diameter ratio (a total length of $55 \mathrm{~mm}$ for a $5 \mathrm{~mm}$ diameter), the wavedispersion must be considered in the equations [5]. The indent is seen as a 1D elastic medium, and the velocity and force determinations from the gauge measurements are performed using Lagrange diagrams. The motion equation at stake is then Love's one, including radial inertia [6]

$$
\frac{\partial^{2} u(x, t)}{\partial t^{2}}=c^{2} \frac{\partial^{2} u(x, t)}{\partial x^{2}}+v^{2} \frac{I}{S} \frac{\partial^{4} u(x, t)}{\partial x^{2} \partial t^{2}}
$$

with $x$ the axial position, $t$ the time, $u$ the particular displacement, $c$ the simple tensioncompression elastic wave celerity, $v$ the Poisson's ratio, $I$ the indent's moment of inertia, $S$ its circular section.
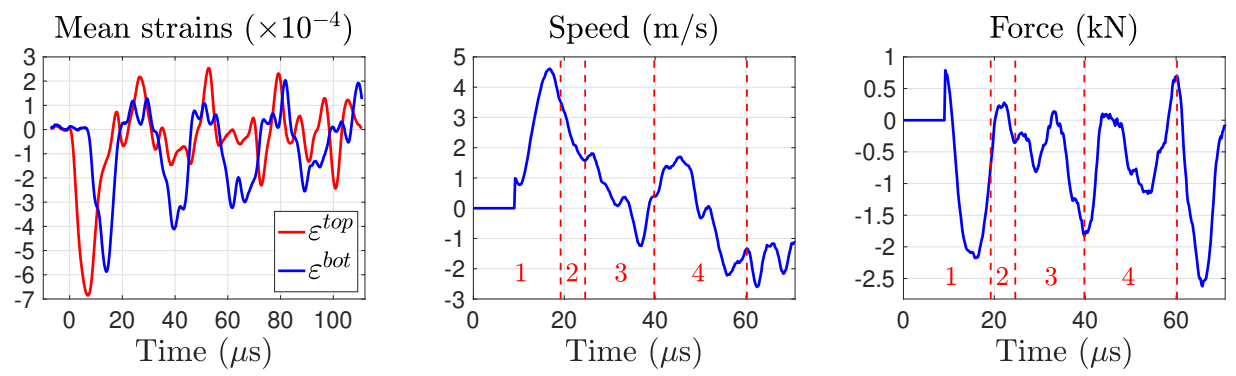

Fig. 2. Gauge data and measured speed and force at the base of the indent cone. Four steps (split by dashed vertical lines) can be distinguished thanks to the fast camera: 1- Dynamic indentation \& elastic phase, in-depth propagation; 2- Star-crack opening; 3- Bouncing; 4- Secondary crack growth.

Gauge measurements are represented on Fig. 2. The speed and force are determined at $1.1 \mathrm{~mm}$ from the glass surface. An initial peak in force and velocity can be noticed at the beginning of impact, at about $15 \mu \mathrm{s}$. If the velocity signal does show a double load, with a second peak at $45.4 \mu \mathrm{s}$, the latter is more difficult to observe on the force curve. The crack formation is also monitored with a Shimadzu ${ }^{\circledR}$ HPV-X2, ultra-high speed camera, with an acquisition rate of 1 to $5 \mathrm{Mfps}$ and a definition of $400 \times 250$ pixels. Thanks to this monitoring, the crack formation is fully captured, and a first crack opening is identified between $19.2 \mu \mathrm{s}$ and $24.6 \mu \mathrm{s}$, followed by a bounce of the indent up to $39.8 \mu \mathrm{s}$. The crack branches secondary growth lasts for $20 \mu \mathrm{s}$. The final crack geometry is reached at $60.2 \mu \mathrm{s}$.

This branches evolution is displayed on Fig. 3. 


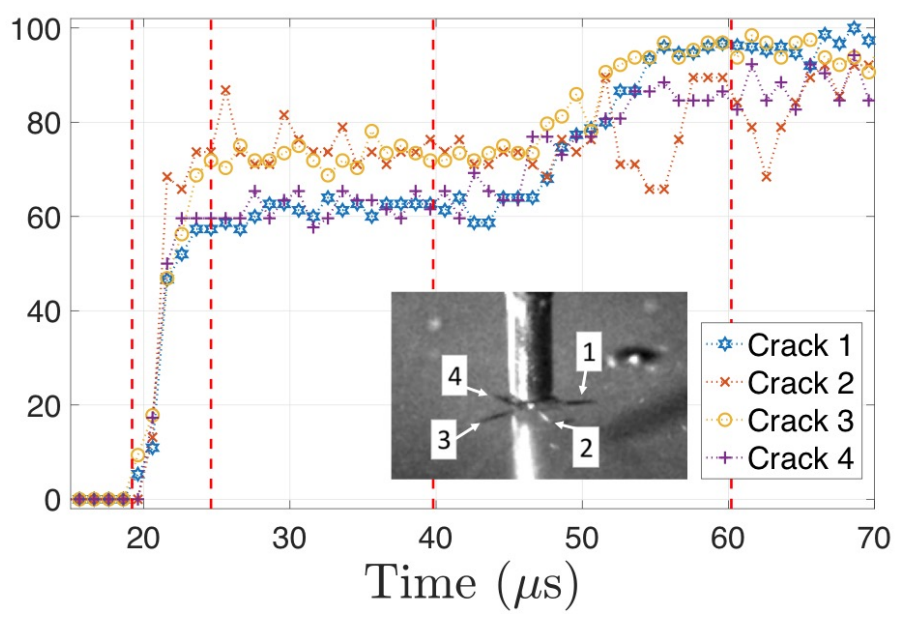

Fig. 3. Crack branches (in $\%$ of total length, $\sim 5 \mathrm{~mm}$ ) evolution versus time. The temporal axis is synchronized regarding the strain gauge measurements with a $0.2 \mu$ s uncertainty.

\section{3 "Cannon" experiments}

The ball drop experiment on laminated glass plates is a certification test detailed in European standards to dissipate impact kinetic energy and to prevent the hazardous ejection of glass fragments.

The "cannon" experiment, whose concept is illustrated on Fig. 4, was designed by Nourry as an instrumented test, which mimics the ball drop experiment with a blunt impactor, but provides quantitative time-resolved measurements of the impactor motion [2]. The various impact speeds allow the observation of different cracking patterns of glass, with a good reproducibility and a large versatility with respect to the laminated glass characteristics (ply thickness, adhesion, pre-damaged surfaces...).

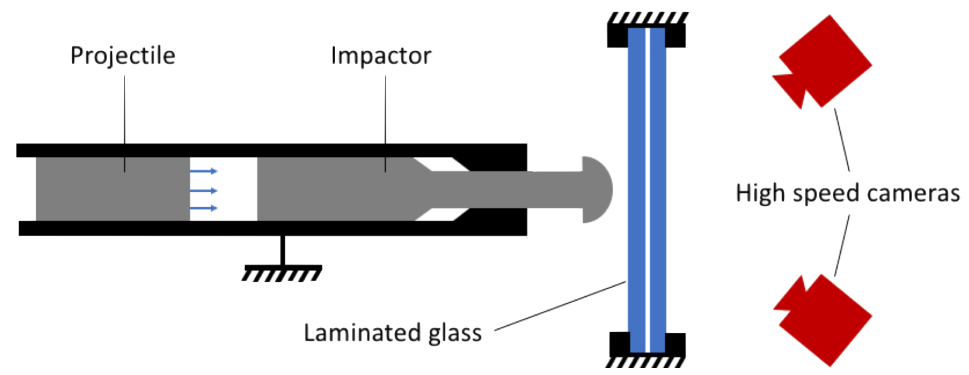

Fig. 4. Scheme of a "cannon" experiment. The impactor and projectile are guided in the barrel. Impactor axial movement is considered.

The mechanical behavior of the interlayer and the mechanics of glass plies are also at stake. 
The high energy dissipation of laminated glass is a subtle balance between two main characteristics: on the one hand, the intrinsic mechanical behavior of the polyvinyl butyral (PVB) interlayer, that can sustain large strains before being torn apart. Then, its engineered adhesion on the glass plies, as delamination enhances the overall deformability of the sample after fragmentation of the glass plies. Despite recent progress on PVB adhesion knowledge for low strain rates [7, 8], the dynamic conditions met in impact experiments remained unexplored. The main purpose of the "cannon" experiments is to link the glass fragmentation, i.e. fragment density and shapes, to the kinematic fields of the laminated glass sample and to the velocity of the $4 \mathrm{~kg}$ impactor. The $300 \mathrm{~mm} \times 300 \mathrm{~mm}$ laminated glass sample is sandwiched between two rings of $140 \mathrm{~mm}$ internal radius and $1 \mathrm{~mm}$ thickness. The plate is not clamped to avoid the glass cracking, but neither rotation, nor bending moment can be neglected on the supporting ring during the experiment.

A telemeter is used to track the impactor movement, giving the half-sphere position during the experiment. The back face of the sample is observed with two Photron ${ }^{\circledR}$ SA-5 high speed cameras which give access to the time history of the impactor head penetration into the assembly, and the temporal sequence of glass fragmentation. On some samples, the back side of the glass is covered with a thin layer of mat elastomeric paint to perform full-field measurements via global stereo-D.I.C. [9]. This technique measures the displacement over the entire speckled area. With two cameras, the 3D surface corresponding to the back face of the glass is evaluated for each step of the experiment.

As certain zones of the speckled surface can not be monitored during the experiment, e.g. zones that are close to the supporting ring, an axisymmetric analysis of the test is proposed, calculating the mean displacement at different radius, then extrapolating the resulting field on the whole plate's surface.

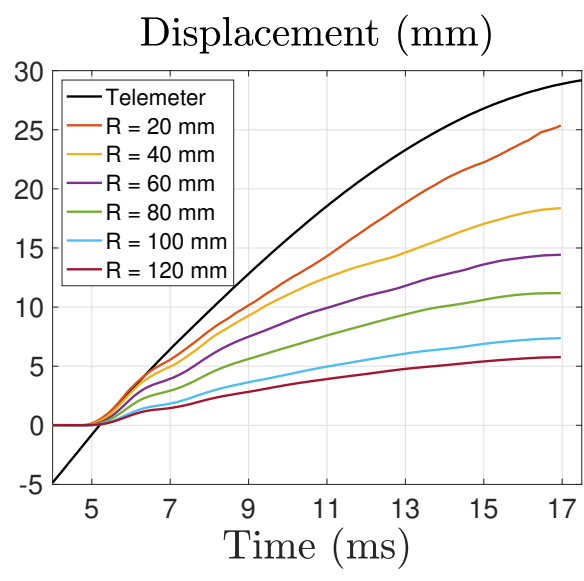

(a)

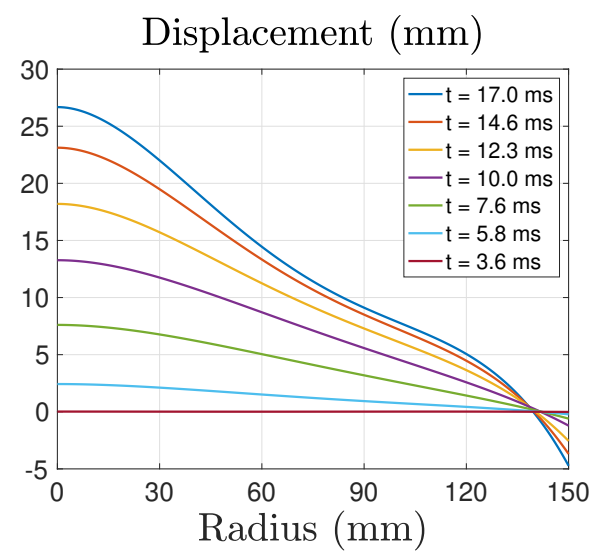

(b)

Fig. 5. Extrapolated out-of-plane displacement measured via axisymmetric stereo-D.I.C.

(a) Out-of-plane displacement versus time, including the displacement measurement by the telemeter (corresponding to $\mathrm{R}=0 \mathrm{~mm}$ ). (b) Out-of-plane displacement extrapolation versus radius. 
The out-of-plane movement, of an experiment with a $4 \mathrm{~m} / \mathrm{s}$ impactor initial velocity, is shown in Fig. 5a. The telemeter curve, corresponding to the impactor displacement, is added to the plot. The deformed surface versus the radius is displayed on Fig. 5b, where extrapolation constraints are illustrated: the imposed displacement at the supporting ring location, i.e. at $140 \mathrm{~mm}$, and the axial symmetry at the center of the plate, i.e. $0 \mathrm{~mm}$.

\section{Conclusion}

Two highly instrumented experimental set-ups designed to study the response of laminated glass to impact were described. The results are promising and pave the way to many new analyses. First, the experimental data combined with a numerical simulation of the sample allow a realistic dynamic mechanical model of laminated glass to be proposed. Next, due to a good reproducibility of the experimental conditions on the two devices, the slightest change on experimental data denotes the influence of glass breakage on the fragmented laminated glass behavior. Varying samples parameters (geometry, adhesion, materials, pre-damaged surfaces...) without changing loading conditions is a great asset to select the best laminated glass specifications. Finally, the residual resistance of a fragmented laminated glass is also at stake, and the well-mastered knowledge of its loading history (e.g. after a first impact) is a huge asset to evaluate the ability to withstand a second impact without perforation.

Acknowledgments: The authors would like to thank Saint-Gobain Research Compiègne for helping carrying out the cannon experiments.

\section{References}

[1] P.V. Grant, W.J. Cantwell, H. McKenzie, P. Corkhill, Int. J. Impact E., 21, pp 737-746 (1998).

[2] E. Nourry, Laminated glass behaviour under perforating impact, $\mathrm{PhD}$ thesis (2005).

[3] R. Decourcelle, Comportement mécanique des vitrages feuilletés sous chargements statiques et dynamiques, $\mathrm{PhD}$ thesis (2011).

[4] H. Kolsky, Proc. Phys. Soc. B, 62, pp 676-700 (1949).

[5] H. Zhao, G. Gary, J. Mech. Phys. Solids, 45, pp 1185-1202 (1997).

[6] M. A. Meyers, Dynamic behavior of materials (Wiley, New York, 1994).

[7] P. Elzière, P. Fourton, Q. Demassieux, A. Chennevière, C. Dalle-Ferrier, C. Creton, M. Ciccotti, E. Barthel, Macromolecules, 52, pp 7821-7830 (2019).

[8] P. Fourton, K. Piroird, M. Ciccotti, E. Barthel, Glass Struct. \& Eng., 5, pp 397-410 (2020).

[9] F. Hild, S. Roux, In Optical Methods for Solid Mechanics. A Full-Field Approach, pp 183-228 (Wiley, Weinheim Germany, 2012). 